\title{
$D$-Stratification and Hierarchy Graphs of the Space of Order 2 and 3 Matrix Pencils
}

\author{
M. I. García-Planas ${ }^{1 *}$ and M. D. Magret ${ }^{1}$ \\ ${ }^{1}$ Departament de Matemàtiques, Universitat Politècnica de Catalunya, Barcelona, Spain \\ * Corresponding author
}

\begin{abstract}
Article Info
Keywords: Equivalence relation, Kronecker normal form, Linear systems, Matrix pencils, Stratification.

2010 AMS: 15A21, 15A22, 93B10, 93B35,

Received: 22 August 2018

Accepted: 2 February 2019

Available online: 20 March 2019
\end{abstract}

\begin{abstract}
Small changes in the entries of a matrix pencil may lead to important changes in its Kronecker normal form. Studies about the effect of small perturbations have been made when considering the stratification associated with the strict equivalence between matrix pencils. In this work, we consider a partition in the space of pairs of matrices associated to regular matrix pencils, which will be proved to be a finite stratification of the space of such matrix pencils, called D-stratification. Matrix pencils in the same strata are those having some prescribed Segre indices. We study the effect of perturbations which lead to changes in the Kronecker canonical form, preserving the order of the nilpotent part. Our goal is to determine which $D$-strata can be reached. In the cases where the order of the matrix pencils is 2 or 3 , we obtain the corresponding hierarchy graphs, illustrating the $D$-strata that can be reached when applying some small perturbations.
\end{abstract}

\section{Introduction}

Jordan normal form of a square matrix $A$ is not stable under small perturbations, small changes in its entries may change the Jordan normal form of the matrix. In [1, 2], V. I. Arnold identified nearby canonical structures using miniversal deformations. H. den Boer and G. Ph. A. Thijsse, A. S. Markus and E. E. Parilis (see [3, 4]) found Jordan normal form of matrices which could be obtained from a given Jordan matrix by arbitrary small perturbations. The changes in the normal form of a matrix when only elements in some concrete positions can be changed are studied by different authors for example, (see [5, 6]).

An informal introduction to perturbations of matrices up to different equivalence relations is given in [7]. Changes of the canonical form for order two and three matrices under congruence were given in [8].

V. I. Arnold introduced the sets of matrices having the same Segre characteristics (and differing only in the continuous invariants) as bundles of matrices. Gibson proved in [9] that this partition is actually a Whitney stratification, the closure of each stratum being the union of strata (of strictly lower dimension).

In [10], [11] a different stratification of the space of square matrices is considered, being the matrices in the same stratum those having the same Drazin inverse (see [12] for this relation between matrices).

The stratification of the space of pairs of matrices related to linear control systems can be found in [13], where a proof of this stratification being Whitney regular in a particular case is included (the general case is an open problem). Bifurcation diagrams were obtained in [14]. Later, the same author proved that the partition of the space of quadruples of matrices according to the set of discrete structural invariants is a stratification (see [15]). All possible Kronecker canonical forms of matrix pencils in a neighbourhood of any given pencil were described by Pokrzywa in [16].

Stratifications can be represented by hierarchy graphs, the nodes being the strata and the edges the covering relations; that is to say, the possible paths from one bundle to another one.

The closure of a stratum consists of all those strata which can be reached applying a small perturbation. Closure relations for matrices under conjugation and matrix pencils under strict equivalence were studied in [17, 18]. In [19] Hasse diagrams for the closure ordening for order two matrices under *congruece were constructed. E. Elmroth, P. Johansson and B. Kågström presented in [18] Stratigraph, a Java-based tool for the computation and visualization of canonical information and stratification hierarchies for matrices and matrix pencils. 
Closure relations for matrix pencils under strict equivalence were studied in [20] and [14]. All possible Kronecker canonical forms of matrix pencils in a neighbourhood of any given matrix pencil were described by Pokrzywa in [16].

As usual, we will denote by $M_{n}(\mathbb{C})$ the set of square matrices of order $n$ with coefficients in $\mathbb{C}$ and by $G l_{n}(\mathbb{C})$ the set of all invertible matrices of order $n$.

\section{Motivation}

Let us consider a linear control dynamical system

$$
E \dot{x}(t)=A x(t)+B u(t)
$$

with $E, A$ square matrices of order n.

In the case where $E$ is an invertible matrix, we can pre-multiply the equation above by $E^{-1}$, thus obtaining:

$$
\dot{x}(t)=A_{1} x(t)+B_{1} u(t)
$$

If $E$ is not invertible, and assuming that the matrix pencil $\lambda E+A$ is regular (to ensure the system has a unique solution) the system splits into a slow and fast subsystems, according to the response's speed to the changes in the control input. These subsystems can be obtained using the Kronecker normal form of the matrix pencil (or its Weierstraß normal form, since we only consider regular matrix pencils). It can be found, for example, in [21]. Let us recall it, we have assumed the matrix pencil $\lambda E+A$ to be regular, there exist invertible matrices $P, Q$ such that

$$
Q(\lambda E+A) P=\lambda\left(\begin{array}{cc}
I_{n_{0}} & 0 \\
0 & \mathscr{N}_{E}
\end{array}\right)+\left(\begin{array}{cc}
G & 0 \\
0 & I_{n-n_{0}}
\end{array}\right)
$$

with $\mathscr{N}_{E}$ a nilpotent matrix, $G \in M_{n_{0}}(\mathbb{C})$ a matrix in Jordan reduced form,

$$
G=\left(\begin{array}{ll}
J & \\
& N_{G}
\end{array}\right)
$$

where $\operatorname{det}(J) \neq 0$ and $N_{G}$ is a nilpotent matrix.

The Kronecker reduced form of the matrix pencil $\lambda E+A$, or Weierstraß form, is:

$$
\begin{aligned}
\lambda E_{c}+A_{c} & =\lambda\left(\begin{array}{cc}
I_{n_{0}} & 0 \\
0 & \mathscr{N}_{E}
\end{array}\right)+\left(\begin{array}{cc}
G & 0 \\
0 & I_{n-n_{0}}
\end{array}\right) \\
& =\lambda\left(\begin{array}{ccc}
I_{V} & 0 & 0 \\
0 & I_{n_{0}-v} & 0 \\
0 & 0 & \mathscr{N}_{E}
\end{array}\right)+\left(\begin{array}{ccc}
J & 0 & 0 \\
0 & N_{G} & 0 \\
0 & 0 & I n-n_{0}
\end{array}\right)
\end{aligned}
$$

Applying suitable basis change and pre-multiplication to the systems' equation (2.1)

$$
E \dot{x}(t)=A x(t)+B u(t)
$$

the system splits into two subsystems:

$$
\left.\begin{array}{rl}
\left(\begin{array}{cc}
I_{v} & 0 \\
0 & I_{n_{0}-v}
\end{array}\right) \dot{x}_{1}(t) & =\left(\begin{array}{cc}
J & 0 \\
0 & N_{G}
\end{array}\right) x_{1}(t)+\left(\begin{array}{l}
B_{1}^{1} \\
B_{1}^{2}
\end{array}\right) u(t) \\
\mathscr{N}_{E} \dot{x}_{2}(t) & =x_{2}(t)+B_{2} u(t)
\end{array}\right\}
$$

The first system is referred to as the slow subsystem and the second one as the fast subsystem. We will denote them by $\Sigma_{S}$ and $\Sigma_{F}$, respectively. The solution of the fast subsystem is well-known (see [22], 1989). Obviously, if E is an invertible matrix, the fast subsystem does not appear $\left(n_{0}=n\right)$.

In turn, the slow subsystem $\Sigma_{S}$ splits into two subsystems:

$$
\left.\begin{array}{lll}
\Sigma_{J} & \dot{y}_{(}(t) & =J y(t)+B_{1}^{1} u(t) \\
\Sigma_{N_{G}} & \dot{z}_{(}(t) & =N_{G} z(t)+B_{1}^{2} u(t)
\end{array}\right\}
$$

That is to say, the initial system can be divided into three independent subsystems:

$$
\left.\begin{array}{lrl}
\Sigma_{J} & \dot{y}_{(}(t) & =J y(t)+B_{1}^{1} u(t) \\
\Sigma_{N_{G}} & \dot{z}_{(}(t) & =N_{G} z(t)+B_{1}^{2} u(t) \\
\Sigma_{F} & \mathscr{N}_{E} \dot{x}_{2}(t) & =x_{2}(t)+B_{2} u(t)
\end{array}\right\}
$$


The solutions to the subsystems above are:

$$
\begin{aligned}
y(t) & =e^{J t} y_{0}+\int_{0}^{t} e^{J(t-\tau)} B_{1}^{1} u(\tau) d \tau \\
z(t) & =e^{N_{G} t} z_{0}+\int_{0}^{t} e^{N(t-\tau)} B_{1}^{2} u(\tau) d \tau \\
& =\sum_{i=0}^{n_{0}-v-1} \frac{1}{i !} N_{G}^{i} t^{i} z_{0}+\sum_{i=0}^{n_{0}-v-1} \frac{1}{i !} \int_{0}^{t} N_{G}^{i} t^{i} B_{1}^{2} u(\tau) d \tau, \\
x_{2} & =-\sum_{i=1}^{n-n_{0}-1} \mathscr{N}^{i} B u^{(i)}(t)
\end{aligned}
$$

and while the solution of the first subsystem, $\Sigma_{J}$ is a matrix series, the solution of the second and third ones, $\Sigma_{N_{G}}$ and $\Sigma_{F}$ are polynomial matrices. The first one depends on the integral of control function and the second one on the derivatives of this function. This supposes an important difference when computing solutions (recall that the different methods and algorithms to compute the exponential of a matrix $J$ are not absolutely satisfactory). On the other hand, the formula to compute the exponential of the matrix is the same independently of the exact value of the eigenvalues and relies only on the Segre characteristics of the matrix.

This suggests to considering a partition in the space of regular matrix pencils, which will be called $D$-stratification, where strata will consist of matrix pencils having the same Segre characteristics in matrix $J$ (and not taking into account the Segre characteristics of matrices $N_{G}$ and $\left.\mathscr{N}_{E}\right)$.

\section{3. $D$-Partition of the space of pairs of matrices associated to regular matrix pencils}

We will denote by $\mathscr{X}$ the set of regular matrix pencils $\lambda E+A$, with $E, A \in M_{n}(\mathbb{C})$ (which is an open subset of the space of pencils of matrices identified with the space of pair of matrices $M_{n}(\mathbb{C}) \times M_{n}(\mathbb{C})$, thus a differentiable manifold).

We define an equivalence relation in $\mathscr{X}$ according to the Segre characteristic of matrix $J$ in the Kronecker reduced form of the matrix pencil $\lambda E+A$.

Definition 3.1. Given two regular matrix pencils $\lambda E+A \in \mathscr{X}, \lambda E^{\prime}+A^{\prime} \in \mathscr{X}$ with Kronecker reduced forms

$$
\begin{aligned}
\lambda E_{c}+A_{c} & =\lambda\left(\begin{array}{ccc}
I_{V} & 0 & 0 \\
0 & I_{n_{0}-v} & 0 \\
0 & 0 & \mathscr{N}_{E}
\end{array}\right)+\left(\begin{array}{ccc}
J & 0 & 0 \\
0 & N_{G} & 0 \\
0 & 0 & I_{n-n_{0}}
\end{array}\right) \\
\lambda E^{\prime}{ }_{c}+A^{\prime}{ }_{c} & =\lambda\left(\begin{array}{cccc}
I_{V^{\prime}} & 0 & 0 \\
0 & I_{n^{\prime}-\nu^{\prime}} & 0 \\
0 & 0 & \mathscr{N}^{\prime}{ }_{E}
\end{array}\right)+\left(\begin{array}{ccc}
J^{\prime} & 0 & 0 \\
0 & N_{G^{\prime}}^{\prime} & 0 \\
0 & 0 & I_{n-n_{0}^{\prime}}
\end{array}\right)
\end{aligned}
$$

they will be said to be D-equivalent if $v=v^{\prime}, n_{0}=n^{\prime}{ }_{0}$ and $J$ and $J^{\prime}$ have the same Segre characteristics.

Note that equivalent pencils in the same orbit under classical equivalent relation of matrix pencils (see [23]) are equivalent under $D$ equivalence relation considered. Therefore equivalent classes are the union of orbits. But matrix pencils in the same stratum of the stratification induced for classical equivalence relation are not necessarily in the same orbit under $D$-equivalence.

Example 3.2. The following matrix pencils

$$
\begin{aligned}
& \lambda E_{1}+A_{1}=\left(\begin{array}{lll}
1 & & \\
& 1 & \\
& & 0
\end{array}\right)+\left(\begin{array}{lll}
2 & & \\
& 3 & \\
& & 1
\end{array}\right) \\
& \lambda E_{2}+A_{2}=\left(\begin{array}{lll}
1 & & \\
& 1 & \\
& & 0
\end{array}\right)+\left(\begin{array}{lll}
2 & & \\
& 0 & \\
& & 1
\end{array}\right)
\end{aligned}
$$

are in the same stratum when considering the stratification induced for classical equivalence (strict equivalence) relation but they are not in the same orbit under D-equivalence.

Equivalent classes under $D$-equivalence relation can be obtained dividing classical strata.

Example 3.3. Let $S$ be the classical stratum consisting of matrix pencils with canonical reduced form

$$
\lambda\left(\begin{array}{cccc}
1 & & & \\
& \ddots & & \\
& & 1 & \\
& & & \mathscr{N}_{E}
\end{array}\right)+\left(\begin{array}{llll}
\lambda_{1} & & & \\
& \ddots & & \\
& & \lambda_{n_{0}} & \\
& & & I_{n-n_{0}}
\end{array}\right)
$$

where $\lambda_{i} \neq \lambda_{j}$ if $i \neq j, 1 \leq i, j \leq n$.

This stratum splits into the following D-equivalence classes: 


$$
\begin{aligned}
& \lambda\left(\begin{array}{cccc}
1 & & & \\
& \ddots & & \\
& & 1 & \\
& & & \mathscr{N}_{E}
\end{array}\right)+\left(\begin{array}{cccc}
\lambda_{1} & & & \\
& \ddots & & \\
& & \lambda_{n_{0}} & \\
& & & I_{n-n_{0}}
\end{array}\right), \quad \quad \lambda_{i} \neq 0, \lambda_{i} \neq \lambda_{j} \text { if } i \neq j \\
& \lambda\left(\begin{array}{ccccc}
1 & & & & \\
& \ddots & & & \\
& & 1 & & \\
& & & 1 & \\
& & & & \mathscr{N}_{E}
\end{array}\right)+\left(\begin{array}{ccccc}
\lambda_{1} & & & & \\
& \ddots & & & \\
& & \lambda_{n_{0}-1} & & \\
& & & 0 & \\
& & & & I_{n-n_{0}}
\end{array}\right), \quad \lambda_{i} \neq 0, \lambda_{i} \neq \lambda_{j} \text { if } i \neq j
\end{aligned}
$$

In order to obtain all the strata, we can proceed as follows. First of all we divide each stratum on the finite classical stratification into a finite number of equivalent classes, separating the orbits in the stratum having some zero-eigenvalue Jordan block and then we joint the sets having the same Segre characteristic correspoding to non-singular part of the Jordan matrix.

Example 3.4. Let us consider the classical strata corresponding to

$$
\begin{aligned}
& \lambda E_{1}+A_{1}=\lambda\left(\begin{array}{ccccc}
1 & & & & \\
& 1 & & & \\
& & 1 & & \\
& & & 0 & 1 \\
& & & & 1
\end{array}\right)+\left(\begin{array}{lllll}
\lambda 1 & & & \\
& \lambda_{2} & 1 & & \\
& & \lambda_{2} & & \\
& & & 1 & \\
& & & & 1
\end{array}\right), \lambda_{1} \neq \lambda_{2} \\
& \lambda E_{2}+A_{2}=\lambda\left(\begin{array}{ccccc}
1 & & & \\
& 1 & & & \\
& & 1 & & \\
& & & 0 & \\
& & & & 0
\end{array}\right)+\left(\begin{array}{lllll}
\lambda 1 & & & \\
& \lambda_{2} & 1 & \\
& & \lambda_{2} & & \\
& & & 1 & \\
& & & & 1
\end{array}\right), \lambda_{1} \neq \lambda_{2}
\end{aligned}
$$

The stratum corresponding to $\lambda E_{1}+A_{1}$ may be divided into

$$
\begin{gathered}
\lambda E_{1}^{1}+A_{1}^{1}=\lambda\left(\begin{array}{ccccc}
1 & & & & \\
& 1 & & & \\
& & 1 & & \\
& & & 0 & 1 \\
& & & & 0
\end{array}\right)+\left(\begin{array}{ccccc}
\lambda_{1} & & & \\
& 0 & 1 & & \\
& & 0 & & \\
& & & 1 & \\
& & & & 1
\end{array}\right), \lambda_{1} \neq 0 \\
\lambda E_{1}^{2}+A_{1}^{2}=\lambda\left(\begin{array}{ccccc}
1 & & & & \\
& 1 & & & \\
& & 1 & & \\
& & & 0 & 1 \\
& & & & 1
\end{array}\right)+\left(\begin{array}{ccccc}
\lambda_{1} & 1 & & \\
& \lambda_{1} & & \\
& & 0 & & \\
& & & 1 & \\
& & & & 1
\end{array}\right), \lambda_{1} \neq 0
\end{gathered}
$$

The stratum corresponding to $\lambda E_{2}+A_{2}$ may be divided into

$$
\begin{gathered}
\lambda E_{2}^{1}+A_{2}^{1}=\lambda\left(\begin{array}{ccccc}
1 & & & & \\
& 1 & & & \\
& & 1 & & \\
& & & 0 & \\
& & & & 0
\end{array}\right)+\left(\begin{array}{ccccc}
\lambda_{1} & & & \\
& 0 & 1 & & \\
& & 0 & & \\
& & & 1 & \\
& & & & 1
\end{array}\right), \lambda_{1} \neq 0 \\
\lambda E_{2}^{2}+A_{2}^{2}=\lambda\left(\begin{array}{ccccc}
1 & & & & \\
& 1 & & & \\
& & 1 & & \\
& & 0 & \\
& & & 0
\end{array}\right)+\left(\begin{array}{ccccc}
\lambda_{1} & 1 & & \\
& \lambda_{1} & & \\
& & 0 & & \\
& & & 1 & \\
& & & & 1
\end{array}\right), \lambda_{1} \neq 0
\end{gathered}
$$

Then, we joint

$$
\left(\lambda E_{1}^{1}+A_{1}^{1}\right) \cup\left(\lambda E_{2}^{1}+A_{2}^{1}\right), \quad\left(\lambda E_{1}^{2}+A_{1}^{2}\right) \cup\left(\lambda E_{2}^{2}+A_{2}^{2}\right)
$$

That is to say, $\lambda E_{1}^{1}+A_{1}^{1}$ and $\lambda E_{2}^{1}+A_{2}^{1}$ are in the same stratum (and all D-equivalent to both pencils) and $\lambda E_{1}^{2}+A_{1}^{2}$ and $\lambda E_{2}^{2}+A_{2}^{2}$ are in the same stratum (and all D-equivalent to both pencils).

Observe that the equivalent classes of $\lambda E_{2}^{i}+A_{2}^{i}(i=1,2)$ is in the frontier (or boundary) of the equivalent classes of $\lambda E_{1}^{i}+A_{1}^{i}(i=1,2)$ : 


$$
\begin{aligned}
& \lim _{\varepsilon \rightarrow 0} \lambda\left(\begin{array}{ccccc}
1 & & & & \\
& 1 & & & \\
& & 1 & & \\
& & & 0 & \varepsilon \\
& & & & 0
\end{array}\right)+\left(\begin{array}{ccccc}
\lambda_{1} & & & & \\
& 0 & 1 & & \\
& & 0 & & \\
& & & 1 & \\
& & & & 1
\end{array}\right)= \\
& \lambda\left(\begin{array}{lllll}
1 & & & & \\
& 1 & & & \\
& & 1 & & \\
& & & 0 & \\
& & & & 0
\end{array}\right)+\left(\begin{array}{ccccc}
\lambda_{1} & & & & \\
& 0 & 1 & & \\
& & 0 & & \\
& & & 1 & \\
& & & & 1
\end{array}\right) \\
& \lim _{\varepsilon \rightarrow 0} \lambda\left(\begin{array}{ccccc}
1 & & & & \\
& 1 & & & \\
& & 1 & & \\
& & & 0 & \varepsilon \\
& & & & 0
\end{array}\right)+\left(\begin{array}{ccccc}
\lambda_{1} & 1 & & & \\
& \lambda_{1} & & & \\
& & 0 & & \\
& & & 1 & \\
& & & & 1
\end{array}\right)= \\
& \lambda\left(\begin{array}{lllll}
1 & & & & \\
& 1 & & & \\
& & 1 & & \\
& & & 0 & \\
& & & & 0
\end{array}\right)+\left(\begin{array}{ccccc}
\lambda_{1} & 1 & & & \\
& \lambda_{1} & & & \\
& & 0 & & \\
& & & 1 & \\
& & & & 1
\end{array}\right)
\end{aligned}
$$

Proposition 3.5. The partition of $\mathscr{X}$ into D-equivalence classes is a finite partition.

We will denote $D$-equivalence classes as $D\left(v, n_{0}, \sigma\right)$, referring to the orders of matrices $J, G$ and the Segre characteristics of $J$. A deeper study of these sets is made below.

Proposition 3.6. Let $J(\sigma)$ the stratum in $G l_{v}(\mathbb{C})$ under similarity and let $\mathscr{N}$ il $\left(n-n_{0}\right)$ and let $\mathscr{N}$ il $\left(n_{0}-v\right)$ be the smooth manifolds of nilpotent matrices of size $n-n_{0}$ and $n_{0}-v$ respectively. Then, there is a smooth monomorphism from the set $J(\sigma) \times \mathscr{N}$ il $\left(n-n_{0}\right) \times$ $\mathscr{N}$ il $\left(n_{0}-v\right)$ to $M_{n}(\mathbb{C}) \times M_{n}(\mathbb{C})$,

$$
\begin{aligned}
\varphi: J(\sigma) \times \mathscr{N} i l\left(n-n_{0}\right) \times \mathscr{N} i l\left(n_{0}-v\right) & \longrightarrow M_{n}(\mathbb{C}) \times M_{n}(\mathbb{C}) \\
(A, \mathscr{N}, N) & \longrightarrow\left(\left(\begin{array}{lll}
I_{v} & & \\
& I_{n_{0}} & \\
& & \mathscr{N}
\end{array}\right),\left(\begin{array}{lll}
A & & \\
& N & \\
& & I_{n_{n_{0}}}
\end{array}\right)\right)
\end{aligned}
$$

Proof. It is straightforward that $\varphi$ is injective and differentiable.

Corollary 3.7. For each $\mathscr{N} \in \mathscr{N}$ il $\left(n-n_{0}\right)$, the map

$$
\begin{aligned}
\varphi_{\mathscr{N}}: J(\sigma) \times \mathscr{N} \text { il }\left(n_{0}-v\right) & \longrightarrow M_{n}(\mathbb{C}) \times M_{n}(\mathbb{C}) \\
(A, \mathscr{N}, N) & \longrightarrow\left(\left(\begin{array}{lll}
I_{v} & & \\
& I_{n_{0}-v} & \\
& & \mathscr{N}
\end{array}\right),\left(\begin{array}{lll}
A & & \\
& N & \\
& & I_{n-n_{0}}
\end{array}\right)\right)
\end{aligned}
$$

is a smooth monomorphism.

Remark 3.8. The D-equivalent class $D\left(v, n_{0}, \sigma\right)$ is the set of equivalent pairs to $\varphi\left(J(\sigma) \times \mathscr{N} i l_{n-n_{0}} \times \mathscr{N} i l_{n_{0}-v}\right)$

$$
\begin{aligned}
& D\left(v, n_{0}, \sigma\right)= \\
& \left\{(A, B)=Q \varphi(A, \mathscr{N}, N) P \mid(A, \mathscr{N}, N) \in J(\sigma) \times \mathscr{N} i l_{n-n_{0}} \times \mathscr{N} i l_{n_{0}-v}, P, Q \in G l_{n}(\mathbb{C})\right\}
\end{aligned}
$$

and each $D$-equivalence class $D\left(v, n_{0}, \sigma\right)$ is a disjoint union of the sets of equivalent pairs to $\varphi_{\mathscr{N}}\left(J(\sigma) \times \mathscr{N} \times \mathscr{N} i_{n_{0}-v}\right)$. Therefore, as a consequence of [10], $D\left(v, n_{0}, \sigma\right)$ is a disjoint union of differentiable manifolds.

In the following section we will show that $D\left(v, n_{0}, \sigma\right)$ are, actually, differentiable manifolds.

\section{Regularity of strata}

First of all we reasoning that the orbits configuring the strata are complex differentiable submanifolds of the set of matrix pencils $M_{n}(\mathbb{C}) \times M_{n}(\mathbb{C})$.

Since orbits under classical equivalent relation of matrix pencils are orbits under the action of the Lie group $\mathscr{G}=\{(P, Q) \in G l(n ; \mathbb{C}) \times$ $G l(n ; \mathbb{C})\}$ under the $\alpha_{\lambda E+A}$ action:

$$
\begin{aligned}
\alpha_{\lambda E+A}: \mathscr{G} \times M_{n}(\mathbb{C}) & \longrightarrow M_{n}(\mathbb{C}) \times M_{n}(\mathbb{C}) \\
(P, Q) & \longrightarrow \lambda P E Q+P A Q
\end{aligned}
$$


Proposition 4.1. The orbits of matrix pencils under classical equivalent relation are complex differentiable submanifolds of $M_{n}(\mathbb{C}) \times M_{n}(\mathbb{C})$

Proof. Taking into account that $\alpha_{\lambda E+A}$ is a rational map and $\mathscr{G}$ is obviously a constructible set, Chevalley's theorem (see for example [24]) states that $\alpha_{\lambda E+A}(\mathscr{G}=\mathscr{O}(\lambda E+A)$ is also constructible. Then $\mathscr{O}(\lambda E+A)$ has a nonsingular point. Taking into account that given any two points on the orbit there is a diffeomorphism of $M_{n}(\mathbb{C}) \times M_{n}(\mathbb{C})$ preserving the orbit and mapping one onto the other, it follows that every point on the orbit is nonsingular. Hence $\mathscr{O}(\lambda E+A)$ is a complex differentiable manifold.

The orbits can be parameterized by

$$
\begin{aligned}
\alpha_{1}: V & \longrightarrow \mathscr{O}(\lambda E+A) \\
(P, Q) & \longrightarrow \alpha_{1}(P, Q)=\alpha_{\lambda E+A}(P, Q)
\end{aligned}
$$

where $V$ is a submanifold of $\mathscr{G}$ minitransversal to the stabilizer defined as $\operatorname{Stab}(\lambda E+A)=\left\{(P, Q) \mid \alpha_{\lambda E+A}(P, Q)=\lambda E+A\right\}$.

From $\alpha_{1}$ we can construct a local diffeomorphism at $(\lambda E+A, \lambda E+A \in \Gamma \times \mathscr{O}(\lambda E+A)$ which preserves the orbits as follows

$$
\begin{aligned}
\beta: \Gamma \times \mathscr{O}(\lambda E+A) & \longrightarrow M_{n}(\mathbb{C}) \times M_{n}(\mathbb{C}) \\
\left((\lambda E+A)+(\lambda X+Y),\left(\lambda E^{\prime}+A^{\prime}\right)\right. & \longrightarrow \alpha_{(\lambda E+A)+(\lambda X+Y)}\left(\alpha_{1}^{-1}\left(\lambda E^{\prime}+A^{\prime}\right)\right)
\end{aligned}
$$

where $\Gamma$ is a variety transversal to the orbit under strict equivalence of matrix pencils.

To study the regularity of strata, we first reduce the problem to the intersection with a variety $\Gamma$ transversal to the orbits of any element of the $D$-equivalence class. The variety considered in this paper is the miniversal deformation obtained in [23]:

$\Gamma=(\lambda E+A)+\{\lambda X+Y\}$, that has the following form for regular matrix pencils in canonical reduced form:

$$
\left(\lambda\left(\begin{array}{ll}
I & \\
& N
\end{array}\right)+\left(\begin{array}{ll}
J & \\
& I
\end{array}\right)\right)+\left(\lambda\left(\begin{array}{ll}
0 & \\
& X_{N}
\end{array}\right)+\left(\begin{array}{ll}
Y_{J} & \\
& 0
\end{array}\right)\right)
$$

where $N+X_{N}$ and $J+Y_{J}$ are miniversal deformations of square matrices under similarity (for instance, given in [1]).

Remark 4.2. $J+Y_{J}=\left(\begin{array}{ll}J_{1} & \\ & J(0)\end{array}\right)+\left(\begin{array}{cc}Y_{J_{1}} & \\ & Y_{J(0)}\end{array}\right)$.

Proposition 4.3. For this particular variety $\Gamma$ considered above,

a) If $\lambda X+Y \neq \lambda 0+0$ then $(\lambda E+A)+(\lambda X+Y) \notin \mathscr{O}(\lambda E+A)$.

b) $(\lambda E+A)+(\lambda X+Y) \in D\left(v, n_{0}, \sigma\right)$ if and only if $J_{1}+Y_{J_{1}}$ has the same Segre symbol than $J_{1}$, and $J(0)+Y_{J(0)}$ and $N+Y_{N}$ are nilpotent.

Lemma 4.4. Let $\lambda E+A$ be a matrix pencil in $M_{n}(\mathbb{C}) \times M_{n}(\mathbb{C}), \mathscr{O}(\lambda E+A)$ its orbit, $D\left(v, n_{0}, \sigma\right)$ its stratum and $\Gamma$ the variety transversal to the orbit defined in [23]. Then, in a neighborhood of $\lambda E+A, D\left(v, n_{0}, \sigma\right)$ is a complex differentiable submanifold of $\mathscr{X}$ if and only if $D\left(v, n_{0}, \sigma\right) \cap \Gamma$ is

Proof. Assume that $D\left(v, n_{0}, \sigma\right)$ is regular at $\lambda E+A$. Taking into account that $\Gamma$ is transversal to the orbit it is also transversal to $D\left(v, n_{0}, \sigma\right)$. Hence $D\left(v, n_{0}, \sigma\right) \cap \Gamma$ is regular at $\lambda E+A$.

Conversely, assume that $D\left(v, n_{0}, \sigma\right) \cap \Gamma$ is regular at $\lambda E+A$. Considering the local diffeormorphism $\beta$ we have

$$
D\left(v, n_{0}, \sigma\right)=\beta\left(D\left(v, n_{0}, \sigma\right) \cup \Gamma\right) \times \mathscr{O}(\lambda E+A)
$$

locally at $\lambda E+A$. Therefore $D\left(v, n_{0}, \sigma\right)$ is regular at $\lambda E+A$.

Then we can conclude the following result.

Theorem 4.5. The sets of the from $D\left(v, n_{0}, \sigma\right)$ are differentiable submanifolds of $\mathscr{X}$.

Proof. Let $\lambda E+A$ be a regular pencil, $\mathscr{O}(\lambda E+A)$ its orbit and $D\left(v, n_{0}, \sigma\right)$ its stratum. We must prove that $D\left(v, n_{0}, \sigma\right)$ is regular at $\lambda E+A$. Taking into account (as we said before) that given any two points in the orbit there is a diffeomorphism of $M_{n}(\mathbb{C}) \times M_{n}(\mathbb{C})$ preserving the orbit and mapping one onto the other. We consider the pencil in its reduced form.

By 4.4 it suffices to prove that $D\left(v, n_{0}, \sigma\right) \cap \Gamma$ is regular at $\lambda E+A$, for that we consider the following map

$$
\begin{aligned}
\phi: M_{V}(\mathbb{C}) \times M_{n-n_{0}}(\mathbb{C}) \times M_{n_{0}-v}(\mathbb{C}) & \longrightarrow M_{n}(\mathbb{C}) \times M_{n}(\mathbb{C}) \\
(A, B, C) & \longrightarrow \lambda\left(\begin{array}{lll}
I & & \\
& I & \\
& & B
\end{array}\right)+\left(\begin{array}{lll}
A & & \\
& B & \\
& & I
\end{array}\right)
\end{aligned}
$$

that is, clearly, a diffeomorphim such that

$$
\phi\left(S_{s}(J(\sigma)) \cap \Gamma_{s}(J) \times S_{s}\left(\mathscr{N}_{E}\right) \cap \Gamma_{s}\left(\mathscr{N}_{E}\right) \cap \mathscr{N} i l_{n-n_{0}} \times S_{s}\left(N_{G}\right) \cap \Gamma_{s}\left(N_{G}\right) \cap \mathscr{N} i l_{n_{0}-v}\right)=D\left(v, n_{0}, \sigma\right) \cap \Gamma
$$

(where $S_{S}(J(\sigma)), S_{S}\left(\mathscr{N}_{E}\right), S_{S}\left(N_{G}\right)$ are the Segre strata of the square matrices $J(\sigma), \mathscr{N}_{E}$ and $N_{G}$ under similarity and $\Gamma_{s}(J(\sigma)), \Gamma_{s}\left(\mathscr{N}_{E}\right)$ and $\Gamma_{s}\left(N_{G}\right)$ are linear varieties transversal to the Segre orbit of $J(\sigma), \mathscr{N}_{E}$ an $N_{G}$ respectively and hence also transversal to $S_{s}(J(\sigma)), S_{s}\left(\mathscr{N}_{E}\right)$ and $S_{S}\left(N_{G}\right)$ at $J(\sigma), \mathscr{N}_{E}$ and $N_{G}$ respectively)

Following [9], Segre strata are regular so, $S_{S}(J(\sigma)) \cap \Gamma_{s}(J(\sigma)), S_{S}\left(\mathscr{N}_{E}\right) \cap \Gamma_{S}\left(\mathscr{N}_{E}\right)$ and $S_{S}\left(N_{G}\right) \cap \Gamma_{S}\left(N_{G}\right)$ are regular at $J(\sigma), \mathscr{N}_{E}$ and $N_{G}$ respectively, and the proof is completed. 
Proposition 4.6. D-equivalence classes verify the frontier condition. That is to say, each frontier of strata consists of strata of strictly lower dimension.

Corollary 4.7. The partition of $\mathscr{X}$ into sets of the form $D\left(v, n_{0}, \sigma\right)$ constitutes a (finite) stratification of $\mathscr{X}$, which will be called $D$ stratification.

\section{Hierarchy diagrams}

Given a non-standard regular pencil, not all small perturbations but some of them lead to a standard pencil with all the non-zero eigenvalues being different from each other.

Example 5.1. Let us consider

$$
\lambda E+A=\lambda\left(\begin{array}{llll}
1 & & & \\
& 1 & & \\
& & 0 & 1 \\
& & & 0
\end{array}\right)+\left(\begin{array}{llll}
1 & & & \\
& 0 & & \\
& & 1 & \\
& & & 1
\end{array}\right)
$$

The small perturbation

$$
(\lambda E+A)(\varepsilon)=\lambda\left(\begin{array}{cccc}
1 & & & \\
& 1 & & \\
& & \varepsilon_{1} & 1 \\
& & & \varepsilon_{2}
\end{array}\right)+\left(\begin{array}{llll}
1 & & & \\
& \varepsilon_{3} & & \\
& & 1 & \\
& & & 1
\end{array}\right), \forall \varepsilon_{i} \neq 0, i=1,2,3,
$$

is equivalent to

$$
\left(\lambda E_{c}+A_{c}\right)(\varepsilon)=\lambda\left(\begin{array}{cccc}
1 & & & \\
& 1 & & \\
& & 1 & \\
& & & 1
\end{array}\right)+\left(\begin{array}{lllll}
1 & & & \\
& \varepsilon_{3} & & \\
& & \frac{1}{\varepsilon_{1}} & \\
& & & \frac{1}{\varepsilon_{2}}
\end{array}\right) .
$$

But between the initial pencil and the more generic one which was obtained, we can find other matrix pencils as, for example,

$$
(\lambda E+A)(\varepsilon)=\lambda\left(\begin{array}{cccc}
1 & & & \\
& 1 & & \\
& & \varepsilon & 1 \\
& & & 0
\end{array}\right)+\left(\begin{array}{cccc}
1 & & & \\
& 0 & & \\
& & 1 & \\
& & & 1
\end{array}\right), \forall \varepsilon \neq 0
$$

that is equivalent to

$$
\left(\lambda E_{c}+A_{c}\right)(\varepsilon)=\lambda\left(\begin{array}{cccc}
1 & & & \\
& 1 & & \\
& & 1 & \\
& & & 0
\end{array}\right)+\left(\begin{array}{llll}
1 & & & \\
& \frac{1}{\varepsilon} & & \\
& & 0 & \\
& & & 1
\end{array}\right) .
$$

Therefore we are interested in finding all possible types of pencils that we can find in a neighbourhood of a given pencil and in what hierarchic position.

The construction of a hierarchy diagram is based upon two facts. First, the order of matrices $\mathscr{N}_{E}$ and $N_{G}$ in the reduced form can be the same or smaller than the original one when applying a small perturbation. The hierarchy diagram in the case the order of these matrices are the same can be deduced from the hierarchy diagrams in the case where square matrices under similarity are considered.

\begin{tabular}{|c|c|}
\hline$D\left(v, n_{0}, \sigma\right)$ & $\lambda A+B$ \\
\hline$D(0,0,-)$ & $\lambda\left(\rho_{0}\right)+($ \\
\hline$D(0,1,-)$ & $\lambda\left(\begin{array}{ll}1 & 0\end{array}\right)+$ \\
\hline$D(1,1,(1))$ & $\lambda(1)+$ \\
\hline$D(0,2,-)$ & $\lambda\left(\begin{array}{ll}1 & 1\end{array}\right)-$ \\
\hline$D(1,2,(1))$ & $\lambda\left(\begin{array}{ll}1 \\
1\end{array}\right)+$ \\
\hline$D(2,2,(2))$ & $\lambda\left(\begin{array}{ll}1 & 1\end{array}\right)+$ \\
\hline$D(2,2,(1,1))$ & \\
\hline$D(2,2,((1) ;(1)))$ & \\
\hline
\end{tabular}

Taking into account the construction of each stratum we can deduce the hierarchic structure from the stratification induced by classical equivalence, by means of breaking joining equivalent strata and replacing them in the closure hierarchic.

We present the hierarchic closure for $n=2$.

First of all, we show the list of all equivalent classes with a representant of each class. 


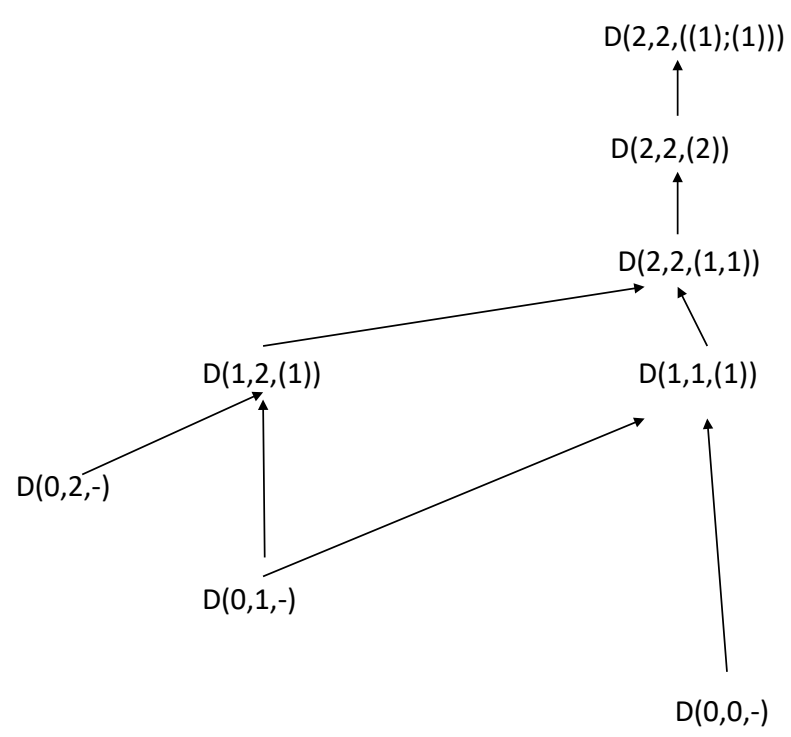

Figure 5.1: Hierarchic closure for $n=2$

Then, the hierarchic closure is given as Figure 5.1.

Notation $D\left(v, n_{0}, \sigma\right) \rightarrow D\left(v^{\prime}, n_{0}^{\prime}, \sigma^{\prime}\right)$ indicates that $D\left(v, n_{0}, \sigma\right) \subset \overline{D\left(v^{\prime}, n_{0}^{\prime}, \sigma^{\prime}\right)}$ where $\overline{D\left(v^{\prime}, n_{0}^{\prime}, \sigma^{\prime}\right)}$ is the closure of $D\left(v^{\prime}, n_{0}^{\prime}, \sigma^{\prime}\right)$.

Now we present the case $n=3$.

As in the case $n=2$ we present the list of all equivalence classes

\begin{tabular}{|c|c|}
\hline$D\left(v, n_{0}, \sigma\right)$ & $\lambda A+B$ \\
\hline$D(0,0,-)$ & $\lambda \rho_{0}^{0}$ \\
\hline$D(0,1,-)$ & \\
\hline$D(1,1,(1))$ & $\lambda(0$ \\
\hline$D(2,2,((1) ;(1)))$ & $\lambda(1$ \\
\hline$D(2,2,(2))$ & \\
\hline$D(2,2,(1,1))$ & \\
\hline$D(1,2,(1))$ & $\lambda($ \\
\hline$D(0,2,-)$ & \\
\hline$D(3,3,((1) ;(1) ;(1)))$ & $\lambda\left(\begin{array}{lll}1 & 1\end{array}\right)$ \\
\hline$D(3,3,((2) ;(1)))$ & $\lambda\left(\begin{array}{ll}1 & \\
& 1\end{array}\right)$ \\
\hline$D(3,3,(3))$ & $\lambda\left(\begin{array}{ll}1 & \\
& 1\end{array}\right)+$ \\
\hline$D(3,3,(2,1))$ & $\lambda\left(\begin{array}{ll}1 & \\
& 1\end{array}\right)+$ \\
\hline$D(3,3,((1,1) ;(1))$ & $\lambda(1$, \\
\hline
\end{tabular}




$$
\begin{aligned}
& D\left(3,3,((1) ;(1) ;(1)) \quad \lambda\left(\begin{array}{lll}
1 & 1 \\
& & 1
\end{array}\right)+\left(\begin{array}{lll}
\lambda & & \\
& \lambda & \\
& & \lambda
\end{array}\right)\right.
\end{aligned}
$$

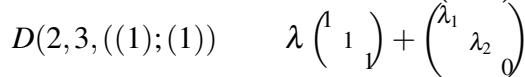

$$
\begin{aligned}
& D(2,3,(2)) \quad \lambda\left(\begin{array}{ll}
1 \\
1
\end{array}\right)+\left(\begin{array}{ll}
\lambda_{1} & 1 \\
& \lambda_{1}
\end{array}\right) \\
& D(2,3,(1,1)) \quad \lambda\left(\begin{array}{lll}
1 & 1 \\
& & 1
\end{array}\right)+\left(\begin{array}{lll}
\lambda_{1} & & \\
& \lambda_{1} & \\
& & 0
\end{array}\right) \\
& D(1,3,(1)) \quad \lambda\left(\begin{array}{lll}
1 & 1 \\
& 1 & 1
\end{array}\right)+\left(\begin{array}{ll}
\lambda_{1} & \\
& 0
\end{array}\right) \\
& D(0,3,-) \quad \lambda\left(\begin{array}{ll}
1 & \\
1 & 1
\end{array}\right)+\left(\begin{array}{ll}
0 \\
0
\end{array}\right)
\end{aligned}
$$

Then, the hierarchic closure is given in figure 5.2.

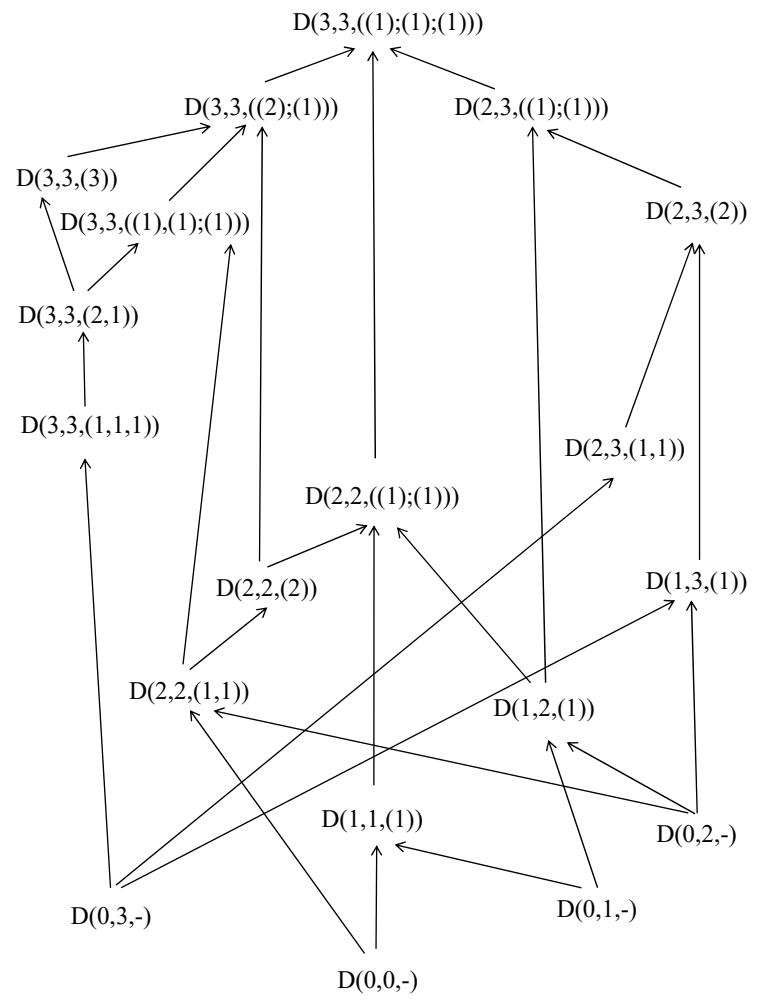

Figure 5.2: Hierarchic closure for $n=3$

Notation $D\left(v, n_{0}, \sigma\right) \rightarrow D\left(v^{\prime}, n_{0}^{\prime}, \sigma^{\prime}\right)$ indicates that $D\left(v, n_{0}, \sigma\right) \subset \overline{D\left(v^{\prime}, n_{0}^{\prime}, \sigma^{\prime}\right)}$ where $\overline{D\left(v^{\prime}, n_{0}^{\prime}, \sigma^{\prime}\right)}$ is the closure of $D\left(v^{\prime}, n_{0}^{\prime}, \sigma^{\prime}\right)$.

\section{Conclusion}

In this work, a partition called D-stratification, in the space of pairs of matrices associated to regular matrix pencils preserving the order of the nilpotent parts has been considered and it was proved to be is a finite stratification of the space of such matrix pencils. This study shows the effect of perturbations over the Kronecker canonical form of a prescribed pencil. We present the $D$-strata that can be reached in the cases where the order of the matrix pencils is 2 or 3 and obtain the corresponding hierarchy graphs, thus illustrating the $D$-strata that is possible to reach when applying small perturbations.

\section{References}

[1] V. I. Arnold, On matrices depending on parameters, Russian Math. Surveys, 26(2), (1971), $29-43$.

[2] V. I. Arnold, Singularity Theory, Cambridge University Press, 1981.

[3] H. den Boer, G. Ph. A. Thijsse, Semi-stability of sums of partial multiplicities under additive perturbation, Integral Equations Operator Theory, 3 (1980), 23-42.

[4] A. S. Markus, E. E. Parilis, The change of the Jordan structure of a matrix under small perturbations, Linear Algebra and its Applications, 54, (1983), $139-152$.

[5] M. A. Beitia, I. de Hoyos, I. Zaballa, The change of similarity invariants under row perturbations: Generic cases, Linear Algebra Appl., 429 (2008), $482-496$. 
[6] M. A. Beitia, I. de Hoyos, I. Zaballa, The change of similarity invariants under row perturbations, Linear Algebra Appl., 429(5-6) (2008), 1302-1333.

[7] L. Klimenko, V.V. Sergeichuk, An informal introduction to perturbations of matrices determined up to similarity or congruence, Sao Paulo Journal of Mathematical Sciences, 8(1), (2014), 1-22.

[8] A. Dmytryshyn, V. Futorny, B. Kågström, L. Klimenko, V.V. Sergeichuk, Change of the congruence canonical form of 2-by-2 and 3-by-3 matrices under perturbations and bundles of matrices under congruence, Linear Algebra Appl., 469 (2015), 305-334.

[9] C. G. Gibson, Regularity of the Segre Stratification, Math. Proc. Camb. Phil. Soc. 80, (1976), 91-97.

[10] M. D. Magret, Stratification of the space of matrices defining linear dynamical systems, Int. J. Complex Systems in Science, 6(1), (2016), 29-35.

[11] M. D. Magret, Perturbation of matrices preserving the Segre characteristics of the non-nilpotent Jordan blocks, CEDYA 2017 - Communications Book, (2017), 429-432.

[12] J. Clotet, M. D. Magret, Familias diferenciables de inversas de Drazin, UPC Commons, 2005.

[13] M. I. García-Planas, Estudio geométrico de familias diferenciables de parejas de matrices: deformaciones versales, cambios de base globales, estratificación de Brunvsky. Ph D. Thesis, UPC, 1994.

[14] M. I. García-Planas, V.V. Sergeichuk, Generic families of matrix pencils and their bifurcation diagrams, Linear Algebra and its Applications 332, (2001), 165-179.

[15] M. I. García-Planas, Kronecker stratification of the space of quadruples of matrices, SIAM Journal on Matrix Analysis and Applications, 19(4), (1998), $872-885$.

[16] A. Pokrzywa, On perturbations and the equivalence orbit of a matrix pencil, Linear Algebra and its Applications, 82, (1986), 99-121.

[17] A. Edelman, E. Elmroth, B. Kågström, A geometric approach to perturbation theory of matrices and matrix pencils. Part II: A stratification-enhanced staircase algorithm, SIAM J. Matrix Anal. Appl., 31(3) (1999), 667-699.

[18] E. Elmroth, P. Johansson, B. Kågström, Computation and presentation of graphs displaying closure hierarchies of Jordan and Kronecker structures, Numer. Linear Algebra Appl., 8(6-7) (2001), 381-399.

[19] V. Futorny, L. Klimenko, V.V. Sergeichuk, Change of the congruence canonical form of 2-by-2 matrices under perturbations, Electron. J. Linear Algebra, 27, (2014), 146-154.

[20] A. Edelman, E. Elmroth, B. Kågström, A geometric approach to perturbation theory of matrices and matrix pencils, Part I: Versal deformations, SIAM J. Matrix Anal. Appl., 18(3) (1997), 653-692.

[21] F. R. Gantmacher, The Theory of Matrices, Chelsea, New York (1959)

[22] L. Dai. Singular Control Systems, Springer Verlag, New York, 1989.

[23] M. I. García-Planas, V.V. Sergeichuk, Simplest miniversal deformations of matrices, matrx pencils, and contragredient matrix pencils, Linear Algebra and its Applications, 302/303, (1999), 45-61.

[24] J. E. Humphreys, Linear Algebraic Groups, Springer-Verlag, (1981). 